\title{
Measure of noncompactness for nonlinear Hilfer fractional differential equation with nonlocal Riemann-Liouville integral boundary conditions in Banach spaces
}

\author{
Abdelatif Boutiara' ${ }^{1}$, Maamar Benbachir ${ }^{2, *}$ and Kaddour Guerbati ${ }^{1}$ \\ 1 Laboratoire de Mathématiques et Sciences Appliquées, University of Ghardaia, Algeria. \\ 2 Faculty of Sciences, Saad Dahlab University, Blida, Algeria. \\ * Correspondence: mbenbachir2001@gmail.com
}

Received: 7 April 2020; Accepted: 28 November 2020; Published: 16 December 2020.

\begin{abstract}
This paper investigates the existence results and uniqueness of solutions for a class of boundary value problems for fractional differential equations with the Hilfer fractional derivative. The reasoning is mainly based upon Mönch's fixed point theorem associated with the technique of measure of noncompactness. We illustrate our main findings, with a particular case example, included to show the applicability of our outcomes. The boundary conditions introduced in this work are of quite general nature and reduce to many special cases by fixing the parameters involved in the conditions.
\end{abstract}

Keywords: Fractional differential equation, Hilfer fractional derivative, nonlocal, Kuratowski measures of noncompactness, Mönch fixed point theorems, Banach space.

MSC: Primary 26A33; Secondary 34B25, 34B15.

\section{Introduction}

$\mathbf{F}$ ractional differential equations have recently been applied in various areas of engineering, mathematics, physics and bio-engineering, and other applied sciences [1,2]. For some fundamental results in the theory of fractional calculus and fractional differential equations, we refer the reader to the monographs of Abbas, Benchohra and N'Guérékata [3], Samko, Kilbas and Marichev [4], Kilbas, Srivastava and Trujillo [5] and Zhou [6], the papers by Abbas et al., [7-9] and the references therein.

In 2000, a generalization of derivatives of both Riemann-Liouville and Caputo was given by Hilfer in [1] when he studied fractional time evolution in physical phenomena. He named it as generalized fractional derivative of order $\alpha \in(0,1)$ and a type $\beta \in[0,1]$ which can be reduced to the Riemann-Liouville and Caputo fractional derivatives when $\beta=0$ and $\beta=1$, respectively. Many authors call it the Hilfer fractional derivative. Such derivative interpolates between the Riemann-Liouville and Caputo derivative in some sense. Some properties and applications of the Hilfer derivative are given in $[1,10]$ and references cited therein.

Recently, considerable attention has been given to the existence of solutions of initial and boundary value problems for fractional differential equations with Hilfer fractional derivative; see [1,2,10-14] and the references therein. In [15-18], the measure of noncompactness was applied to some classes of functional Riemann-Liouville or Caputo fractional differential equations in Banach spaces.

In this paper, we consider the existence of solutions of the following boundary value problem for a nonlinear fractional differential equation,

$$
\begin{cases}D_{0^{+}}^{\alpha, \beta} y(t)=f(t, y(t)), & t \in J:=[0, T] \\ a_{1} I^{1-\gamma} y(0)+b_{1} I^{1-\gamma+q_{1}} y\left(\eta_{1}\right)=\lambda_{1}, & 0<q_{1} \leq 1 \\ a_{2} I^{1-\gamma} y(T)+b_{2} I^{1-\gamma+q_{2}} y\left(\eta_{2}\right)=\lambda_{2}, & 0<q_{2} \leq 1 .\end{cases}
$$

where $D_{0^{+}}^{\alpha, \beta}$ is the Hilfer fractional derivative, where $1<\alpha \leq 2,0 \leq \beta \leq 1,0<\eta_{i}<T, \mathrm{i}=1,2, \gamma=\alpha+\beta-\alpha \beta$. Let $E$ is a reflexive Banach space with norm $\|\|, f:. J \times E \times E \times E \rightarrow E$ is given continuous function and satisfying some assumptions that will be specified later and $a_{i}, b_{i}, \lambda_{i}, i=1,2$ are real constants.

The organization of this work is as follows; in Section 2, we introduce some notations, definitions, and lemmas that will be used later. Section 3 treats the existence of solutions in Banach spaces by using the Mönch's 
fixed point theorem combined with the technique of measures of noncompactness. In Section 4, we illustrate the obtained results by an example. Finally, the paper concludes with some interesting observations.

\section{Preliminaries}

In what follows we introduce definitions, notations, and preliminary facts which are used in the sequel. For more details, we refer to [1,4,5,19-22].

Let $C(J, E)$ be the Banach space of continuous functions $y: J \rightarrow E$, with the usual supremum norm

$$
\|y\|_{\infty}=\sup \{\|y(t)\|, t \in J\}
$$

and $L^{1}(J, E)$ be the Banach space of measurable functions $y: J \rightarrow E$ which are Bochner integrable, equipped with the norm

$$
\|y\|_{L^{1}}=\int_{J} y(t) d t
$$

Further, let $A C^{1}(J, E)$ be the space of functions $y: J \rightarrow E$, whose first derivative is absolutely continuous.

Definition 1. [23] Let $J=[0, T]$ be a finite interval and $1 \leq \gamma<2$. We introduce the weighted space $C_{1-\gamma}(J, E)$ of continuous functions $f$ on $(0, T]$ by

$$
C_{1-\gamma}(J, E)=\left\{f:(0, T] \rightarrow E:(t-a)^{1-\gamma} f(t) \in C(J, E)\right\} .
$$

In the space $C_{1-\gamma}(J, E)$, we define the norm

$$
\|f\|_{C_{1-\gamma}}=\left\|(t-a)^{1-\gamma} f(t)\right\|_{C} .
$$

Definition 2. [23] Let $1<\alpha<2,0 \leq \beta \leq 1$. The weighted space $C_{1-\gamma}^{\alpha, \beta}(J, E)$ is defined by

$$
C_{1-\gamma}^{\alpha, \beta}(J, E)=\left\{f:(0, T] \rightarrow \mathbb{R}: D_{0^{+}}^{\alpha, \beta} f \in C_{1-\gamma}(J, E)\right\}, \gamma=\alpha+\beta-\alpha \beta,
$$

and

$$
C_{1-\gamma}^{1}(J, E)=\left\{f:(0, T] \rightarrow \mathbb{R}: f^{\prime} \in C_{1-\gamma}(J, E)\right\}, \gamma=\alpha+\beta-\alpha \beta,
$$

with the norm

$$
\|f\|_{C_{1-\gamma}^{1}}=\|f\|_{C}+\left\|f^{\prime}\right\|_{C_{1-\gamma}}
$$

Moreover, $C_{1-\gamma}(J, E)$ is complete metric space of all continuous functions mapping $J$ into $E$ with the metric $d$ defined by

$$
d\left(y_{1}, y_{2}\right)=\left\|y_{1}-y_{2}\right\|_{C_{1-\gamma}(J, E)}:=\max _{t \in J}\left|(t-a)^{1-\gamma}\left[y_{1}(t)-y_{2}(t)\right]\right| .
$$

For details, see [23].

Now, we give some results and properties of fractional calculus.

Definition 3. [24] Let $(0, T]$ and $f:(0, \infty) \rightarrow \mathbb{R}$ is a real valued continuous function. The Riemann-Liouville fractional integral of a function $f$ of order $\alpha \in \mathbb{R}^{+}$is denoted as $I_{0^{+}}^{\alpha} f$ and defined by

$$
I_{0^{+}}^{\alpha} f(t)=\frac{1}{\Gamma(\alpha)} \int_{0}^{t}(t-s)^{\alpha-1} f(s) d s, \quad t>0,
$$

where $\Gamma(\alpha)$ is the Euler's Gamma function.

Definition 4. [5] Let $(0, T$ ] and $f:(0, \infty) \rightarrow \mathbb{R}$ is a real valued continuous function. The Riemann-Liouville fractional derivative of a function $f$ of order $\alpha \in \mathbb{R}_{0}^{+}=[0,+\infty)$ is denoted as $D_{0^{+}}^{\alpha} f$ and defined by

$$
D_{0^{+}}^{\alpha} f(t)=\frac{1}{\Gamma(n-\alpha)} \frac{d^{n}}{d t^{n}} \int_{0}^{t}(t-s)^{n-\alpha-1} f(s) d s,
$$


where $n=[\alpha]+1$, and $[\alpha]$ means the integral part of $\alpha$, provided the right hand side is pointwise defined on $(0, \infty)$.

Definition 5. [5] The Caputo fractional derivative of function $f$ with order $\alpha>0, n-1<\alpha<n, n \in \mathbb{N}$ is defined by

$$
{ }^{C} D_{0^{+}}^{\alpha} f(t)=\frac{1}{\Gamma(n-\alpha)} \int_{0}^{t}(t-s)^{n-\alpha-1} f^{(n)}(s) d s, \quad t>0 .
$$

In [1], Hilfer studied applications of a generalized fractional operator having the Riemann-Liouville and Caputo derivatives as specific cases, (see also [2,10]).

Definition 6. [1] The Hilfer fractional derivative $D_{0^{+}}^{\alpha, \beta}$ of order $\alpha(n-1<\alpha<n)$ and type $\beta(0 \leq \beta \leq 1)$ is defined by

$$
D_{0^{+}}^{\alpha, \beta}=I_{0^{+}}^{\beta(n-\alpha)} D^{n} I_{0^{+}}^{(1-\beta)(n-\alpha)} f(t),
$$

where $I_{0^{+}}^{\alpha}$ and $D_{0^{+}}^{\alpha}$ are Riemann-Liouville fractional integral and derivative defined by (3) and (4), respectively.

Remark 1. ([25]) Hilfer fractional derivative interpolates between the Riemann-Liouville ((4), if $\beta=0)$ and Caputo ((5), if $\beta=1$ ) fractional derivatives since

$$
D_{0^{+}}^{\alpha, 0}={ }^{R-L} D_{0^{+}}^{\alpha} \text { and } D^{\alpha, 1}={ }^{C} D_{0^{+}}^{\alpha} .
$$

Lemma 1. Let $1<\alpha<2,0 \leq \beta \leq 1, \gamma=\alpha+\beta-\alpha \beta$, and $f \in L^{1}(J, E)$. The operator $D_{0^{+}}^{\alpha, \beta}$ can be written as

$$
D_{0^{+}}^{\alpha, \beta} f(t)=\left(I_{0^{+}}^{\beta(1-\alpha)} \frac{d}{d t} I_{0^{+}}^{(1-\gamma)} f\right)(t)=I_{0^{+}}^{\beta(1-\alpha)} D^{\gamma} f(t), \quad t \in J
$$

Lemma 2. Let $1<\alpha<2,0 \leq \beta \leq 1$ and $\gamma=\alpha+\beta-\alpha \beta$. If $D_{0^{+}}^{\beta(1-\alpha)} f$ exists and is in $L^{1}(J, E)$, then

$$
D_{0^{+}}^{\alpha, \beta} I_{0^{+}}^{\alpha} f(t)=I_{0^{+}}^{\beta(1-\alpha)} D_{0^{+}}^{\beta(1-\alpha)} f(t), \quad t \in J
$$

Furthermore, if $f \in C_{1-\gamma}(J, E)$ and $I_{0^{+}}^{1-\beta(1-\alpha)} f \in C_{1-\gamma}^{1}(J, E)$, then

$$
D_{0^{+}}^{\alpha, \beta} I_{0^{+}}^{\alpha} f(t)=f(t), \quad t \in J
$$

Lemma 3. Let $1<\alpha<2,0 \leq \beta \leq 1, \gamma=\alpha+\beta-\alpha \beta$, and $f \in L^{1}(J, E)$. If $D_{0^{+}}^{\gamma} f$ exists and is in $L^{1}(J, E)$, then

$$
I_{0^{+}}^{\alpha} D_{0^{+}}^{\alpha, \beta} f(t)=I_{0^{+}}^{\gamma} D_{0^{+}}^{\gamma} f(t)=f(t)-\frac{I_{0^{+}}^{1-\gamma} f\left(0^{+}\right)}{\Gamma(\gamma)} t^{\gamma-1}, \quad t \in J .
$$

Lemma 4. [5] For $t>$ a, we have

$$
\begin{cases}I_{0^{+}}^{\alpha}(t-a)^{\beta-1}(t) & =\frac{\Gamma(\beta)}{\Gamma(\beta-\alpha)}(t-a)^{\beta+\alpha-1} \\ D_{0^{+}}^{\alpha}(t-a)^{\beta-1}(t) & =\frac{\Gamma(\beta)}{\Gamma(\beta-\alpha)}(t-a)^{\beta-\alpha-1}\end{cases}
$$

Lemma 5. Let $\alpha>0$ and $0 \leq \beta \leq 1$. Then the homogeneous differential equation with Hilfer fractional order

$$
D_{0^{+}}^{\alpha, \beta} h(t)=0
$$

has a solution

$$
h(t)=c_{0} t^{\gamma-1}+c_{1} t^{\gamma+2 \beta-2}+c_{2} t^{\gamma+2(2 \beta)-3}+\ldots+c_{n} t^{\gamma+n(2 \beta)-(n+1)} .
$$

Notation 1. For a given set $V$ of functions $v: J \rightarrow E$, let us denote by

$$
V(t)=\{v(t): v \in V\}, t \in J,
$$

and

$$
V(J)=\{v(t): v \in V, t \in J\} .
$$


Definition 7. A map $f: J \times E \rightarrow E$ is said to be Caratheodory if

(i) $t \mapsto f(t, u)$ is measurable for each $u \in E$;

(ii) $u \mapsto F(t, u)$ is continuous for almost all $t \in J$.

For convenience, we recall the definitions of the Kuratowski measure of noncompactness and summarize the main properties of this measure.

Definition 8. ([16,19]). Let $E$ be a Banach space and $\Omega_{E}$ the bounded subsets of $E$. The Kuratowski measure of noncompactness is the map $\mu: \Omega_{E} \rightarrow[0, \infty]$ defined by

$$
\mu(B)=\inf \left\{\epsilon>0: B \subseteq \cup_{i=1}^{n} B_{i} \text { and } \operatorname{diam}\left(B_{i}\right) \leq \epsilon\right\} ; \text { here } B \in \Omega_{E} .
$$

This measure of noncompactness satisfies following important properties [16,19]:

(a) $\mu(B)=0 \Leftrightarrow \bar{B}$ is compact ( $B$ is relatively compact).

(b) $\mu(B)=\mu(\bar{B})$.

(c) $A \subset B \Rightarrow \mu(A) \leq \mu(B)$.

(d) $\mu(A+B) \leq \mu(A)+\mu(B)$.

(e) $\mu(c B)=|c| \mu(B) ; c \in \mathbb{R}$.

(f) $\mu(\operatorname{conv} B)=\mu(B)$.

Let us now recall Mönch's fixed point theorem and an important lemma.

Theorem 1. ([15,22]). Let $D$ be a bounded, closed and convex subset of a Banach space such that $0 \in D$, and let $N$ be a continuous mapping of $D$ into itself. If the implication

$$
V=\overline{\operatorname{conv}} N(V) \text { or } V=N(V) \cup\{0\} \Rightarrow \mu(V)=0
$$

holds for every subset $V$ of $D$, then $N$ has a fixed point.

Lemma 6. ([22]). Let $D$ be a bounded, closed and convex subset of the Banach space $C(J, E)$, "G" a continuous function on $J \times J$ and " $f$ " a function from $J \times E \longrightarrow E$ which satisfies the Caratheodory conditions, and suppose there exists $p \in L^{1}\left(J, \mathbb{R}^{+}\right)$such that, for each $t \in J$. Then for each bounded set $B \subset E$, we have

$$
\lim _{h \rightarrow 0^{+}} \mu\left(f\left(J_{t, h} \times B\right)\right) \leq p(t) \mu(B) ; \text { here } J_{t, h}=[t-h, t] \cap J .
$$

If $V$ is an equicontinuous subset of $D$, then

$$
\mu\left(\left\{\int_{J} G(s, t) f(s, y(s)) d s: y \in V\right\}\right) \leq \int_{J}\|G(t, s)\| p(s) \mu(V(s)) d s .
$$

\section{Main results}

Let us start by defining what we meant by a solution of Problem (1).

Definition 9. A function $y \in C_{1-\gamma}(J, E)$ is said to be a solution of the Problem (1) if $y$ satisfies the equation $D_{0^{+}}^{\alpha, \beta} y(t)=f(t, y(t))$ on $J$, and the conditions $a_{1} I^{1-\gamma} y(0)+b_{1} I^{1-\gamma+q_{1}} y\left(\eta_{1}\right)=\lambda_{1}$ and $a_{2} I^{1-\gamma} y(T)+$ $b_{2} I^{1-\gamma+q_{2}} y\left(\eta_{2}\right)=\lambda_{2}$.

Lemma 7. Let $f: J \times E \times E \times E \rightarrow E$ be a function such that $f \in C_{1-\gamma}(J, E)$ for any $y \in C_{1-\gamma}(J, E)$. Then the unique solution of the linear Hilfer fractional boundary value problem

$$
D_{0^{+}}^{\alpha, \beta} y(t)=f(t, y(t)), t \in J:=[0, T]
$$

with boundary conditions

$$
\begin{cases}a_{1} I^{1-\gamma} y(0)+b_{1} I^{1-\gamma+q_{1}} y\left(\eta_{1}\right) & =\lambda_{1} \\ a_{2} I^{1-\gamma} y(T)+b_{2} I^{1-\gamma+q_{2}} y\left(\eta_{2}\right) & =\lambda_{2}, \gamma=\alpha+\beta-\alpha \beta\end{cases}
$$


is given by

$$
\begin{aligned}
y(t)= & I^{\alpha} f(t, y(t))+\frac{t^{\gamma-1}}{w}\left[\left(w_{4} \lambda_{1}-w_{2} \lambda_{2}\right)-w_{4} b_{1} I^{\alpha-\gamma+q_{1}+1} f\left(\eta_{1}, y\left(\eta_{1}\right)\right)\right. \\
& \left.+w_{2}\left(a_{2} I^{\alpha-\gamma+1} f(T, y(T))+b_{2} I^{\alpha-\gamma+q_{2}+1} f\left(\eta_{2}, y\left(\eta_{2}\right)\right)\right)\right] \\
& +\frac{t^{\gamma+2 \beta-2}}{w}\left[\left(w_{1} \lambda_{2}-w_{3} \lambda_{1}\right)+w_{3} b_{1} I^{\alpha-\gamma+q_{1}+1} f\left(\eta_{1}, y\left(\eta_{1}\right)\right)\right. \\
& \left.-w_{1}\left(a_{2} I^{\alpha-\gamma+1} f(T, y(T))+b_{2} I^{\alpha-\gamma+q_{2}+1} f\left(\eta_{2}, y\left(\eta_{2}\right)\right)\right)\right] \\
= & I^{\alpha} f(t, y(t))+\frac{\left(w_{3} t^{\gamma+2 \beta-2}-w_{4} t^{\gamma-1}\right)}{w} b_{1} I^{\alpha-\gamma+q_{1}+1} f\left(\eta_{1}, y\left(\eta_{1}\right)\right)+\frac{t^{\gamma-1}}{w}\left(w_{4} \lambda_{1}-w_{2} \lambda_{2}\right) \\
& +\frac{\left(w_{2} t^{\gamma-1}-w_{1} t^{\gamma+2 \beta-2}\right)}{w}\left(a_{2} I^{\alpha-\gamma+1} f(T, y(T))+b_{2} I^{\alpha-\gamma+q_{2}+1} f\left(\eta_{2}, y\left(\eta_{2}\right)\right)\right) \\
& +\frac{t^{\gamma+2 \beta-2}}{w}\left(w_{1} \lambda_{2}-w_{3} \lambda_{1}\right),
\end{aligned}
$$

where

$$
\begin{cases}w_{1}=\Gamma(\gamma)\left(a_{1}+b_{1} \frac{\eta_{1}^{q_{1}}}{\Gamma\left(q_{1}+1\right)}\right) \\ w_{2}=\frac{\Gamma(\gamma+2 \beta-1)}{\Gamma\left(2 \beta+q_{1}\right)} \eta_{1}^{2 \beta+q_{1}-1}, \\ w_{3}=\Gamma(\gamma)\left(a_{2}+b_{2} \frac{\eta_{2}^{q_{2}}}{\Gamma\left(q_{2}+1\right)}\right), \\ w_{4}=\frac{\Gamma(\gamma+2 \beta-1)}{\Gamma(2 \beta)}\left(b_{2} \eta_{2}^{2 \beta+q_{2}-1}+a_{2} T^{2 \beta-1}\right), \\ w=w_{1} w_{4}-w_{2} w_{3}, \quad \text { with } \quad w \neq 0 .\end{cases}
$$

Proof. Assume $y$ satisfies (12), then Lemma 5 implies that

$$
y(t)=c_{1} t^{\gamma-1}+c_{2} t^{\gamma+2 \beta-2}+\frac{1}{\Gamma(\alpha)} \int_{0}^{t}(t-s)^{\alpha-1} f(s, y(s)) d s,
$$

for some constants $c_{1}, c_{2} \in \mathbb{R}$. Applying the boundary conditions (11) in (14), we obtain

$$
\begin{aligned}
& I^{1-\gamma} y(t)=I^{\alpha-\gamma+1} f(t, y(t))+c_{1} \Gamma(\gamma)+c_{2} \frac{\Gamma(\gamma+2 \beta-1)}{\Gamma(2 \beta)} t^{2 \beta-1}, \\
& I^{1-\gamma} y(0)=c_{1} \Gamma(\gamma) \\
& I^{1-\gamma} y(T)=I^{\alpha-\gamma+1} f(T, y(T))+c_{1} \frac{\Gamma(\gamma)}{\Gamma\left(q_{i}+1\right)}+c_{2} \frac{\Gamma(\gamma+2 \beta-1)}{\Gamma(2 \beta)} T^{2 \beta-1}, \\
& I^{1-\gamma+q_{i}} y\left(\eta_{i}\right)=I^{\alpha-\gamma+q_{i}+1} f\left(\eta_{i}, y\left(\eta_{i}\right)\right)+c_{1} \Gamma(\gamma)+c_{2} \frac{\Gamma(\gamma+2 \beta-1)}{\Gamma\left(2 \beta+q_{i}\right)} \eta_{i}^{2 \beta+q_{i}-1}, i=1,2 .
\end{aligned}
$$

After collecting the similar terms in one part and by using (13), we have the following equations

$$
\left\{\begin{array}{l}
c_{1} w_{1}+c_{2} w_{2}=\lambda_{1}-b_{1} I^{\alpha-\gamma+q_{1}+1} f\left(\eta_{1}, y\left(\eta_{1}\right)\right) \\
c_{1} w_{3}+c_{2} w_{4}=\lambda_{2}-a_{2} I^{\alpha-\gamma+1} f(T, y(T))-b_{2} I^{\alpha-\gamma+q_{2}+1} f\left(\eta_{2}, y\left(\eta_{2}\right)\right) .
\end{array}\right.
$$

Solving (15), we find that

$c_{1}=\frac{1}{w}\left[\left(w_{4} \lambda_{1}-w_{2} \lambda_{2}\right)-w_{4} b_{1} I^{\alpha-\gamma+q_{1}+1} f\left(\eta_{1}, y\left(\eta_{1}\right)\right)+w_{2}\left(a_{2} I^{\alpha-\gamma+1} f(T, y(T))+b_{2} I^{\alpha-\gamma+q_{2}+1} f\left(\eta_{2}, y\left(\eta_{2}\right)\right)\right)\right]$, and

$c_{2}=\frac{1}{w}\left[\left(w_{1} \lambda_{2}-w_{3} \lambda_{1}\right)+w_{3} b_{1} I^{\alpha-\gamma+q_{1}+1} f\left(\eta_{1}, y\left(\eta_{1}\right)\right)-w_{1}\left(a_{2} I^{\alpha-\gamma+1} f(T, y(T))+b_{2} I^{\alpha-\gamma+q_{2}+1} f\left(\eta_{2}, y\left(\eta_{2}\right)\right)\right)\right]$.

Substituting the value of $c_{1}, c_{2}$ in (14), we get (12).

In order to present and prove our main results, we consider the following theorem: 
Theorem 2. Assume that the following conditions hold:

(H1) $f: J \times E \rightarrow E$ satisfies the Caratheodory conditions;

(H2) There exists $p \in L^{1}\left(J, \mathbb{R}^{+}\right)$, such that, $\|f(t, y)\| \leq p(t)\|y\|$, for $t \in J$ and each $y \in E$;

(H3) For each $t \in J$ and each bounded set $B \subset E$, we have $\lim _{h \rightarrow 0^{+}} \mu\left(f\left(J_{t, h} \times B\right)\right) \leq t^{1-\gamma} p(t) \mu(B)$; here $J_{t, h}=$ $[t-h, t] \cap J$

(H4) There exists a constant $R>0$ such that

$$
R \geq \frac{K}{\left(1-p^{*} L\right)},
$$

where $L=\frac{T^{\alpha-\gamma+1}}{\Gamma(\alpha+1)}+\frac{\left|b_{1}\right|\left(\left|w_{3}\right| T^{2 \beta-1}+\left|w_{4}\right|\right)}{w \Gamma\left(\alpha-\gamma+q_{1}+2\right)} \eta_{1}^{\alpha-\gamma+q_{1}+1}+\frac{\left|a_{2}\right|\left(\left|w_{2}\right|+\left|w_{1}\right| T^{2 \beta-1}\right)}{|w| \Gamma(\alpha-\gamma+2)} T^{\alpha-\gamma+1}+\frac{\left|b_{2}\right|\left(\left|w_{2}\right|+\left|w_{1}\right| T^{2 \beta-1}\right)}{|w| \Gamma\left(\alpha-\gamma+q_{2}+2\right)} \eta_{2}^{\alpha-\gamma+q_{2}+1}$. and $K=\frac{T^{2 \beta-1}\left(\left|w_{1} \lambda_{2}\right|+\left|w_{3} \lambda_{1}\right|\right)+\left(\left|w_{4} \lambda_{1}\right|+\left|w_{2} \lambda_{2}\right|\right)}{|w|}$.

Now, we shall prove the following theorem concerning the existence of solutions of (1). Let $p^{*}=$ $\sup _{t \in J} p(t)$.

Theorem 3. Assume that the hypotheses (H1)-(H3) hold. If

$$
p^{*} L<1
$$

then (1) has at least one solution defined on J.

Proof. Transform the Problem (1) into a fixed point problem. Consider the operator $\aleph: C_{1-\gamma}(J, E) \rightarrow$ $C_{1-\gamma}(J, E)$ defined by

$$
\begin{aligned}
& \aleph(y)(t)=I^{\alpha} f(t, y(t))+\frac{\left(w_{3} t^{\gamma+2 \beta-2}-w_{4} t^{\gamma-1}\right)}{w} b_{1} I^{\alpha-\gamma+q_{1}+1} f\left(\eta_{1}, y\left(\eta_{1}\right)\right)+\frac{t^{\gamma-1}}{w}\left(w_{4} \lambda_{1}-w_{2} \lambda_{2}\right) \\
& +\frac{\left(w_{2} t^{\gamma-1}-w_{1} t^{\gamma+2 \beta-2}\right)}{w}\left(a_{2} I^{\alpha-\gamma+1} f(T, y(T))+b_{2} I^{\alpha-\gamma+q_{2}+1} f\left(\eta_{2}, y\left(\eta_{2}\right)\right)\right)+\frac{t^{\gamma+2 \beta-2}}{w}\left(w_{1} \lambda_{2}-w_{3} \lambda_{1}\right) .
\end{aligned}
$$

Clearly, the fixed points of the operator $\aleph$ are solutions of the Problem (1).

Take

$$
D=\left\{y \in C_{1-\gamma}(J, E):\|y\| \leq R\right\}
$$

where $R$ satisfies inequality (16). Notice that the subset $D$ is closed, convex, and equicontinuous. We shall show that the operator $\aleph$ satisfies all the assumptions of Mönch's fixed point theorem. The proof will be given in three steps.

Step 1. $\aleph$ is continuous.

Let $y_{n}$ be a sequence such that $y_{n} \rightarrow y$ in $C_{1-\gamma}(J, E)$. Then for each $t \in J$,

$$
\begin{aligned}
& \left\|t^{1-\gamma}\left(\aleph\left(y_{n}\right)(t)-\aleph(y)(t)\right)\right\| \leq \frac{t^{1-\gamma}}{\Gamma(\alpha)} \int_{0}^{t}(t-s)^{\alpha-1}\left\|f\left(s, y_{n}(s)\right)-f(s, y(s))\right\| d s \\
& +\frac{\left|b_{1}\right|\left(\left|w_{3}\right| t^{2 \beta-1}+\left|w_{4}\right|\right)}{|w| \Gamma\left(\alpha-\gamma+q_{1}+1\right)} \int_{0}^{\eta_{1}}\left(\eta_{1}-s\right)^{\alpha-\gamma+q_{1}}\left\|f\left(s, y_{n}(s)\right)-f(s, y(s))\right\| d s \\
& +\frac{\left|a_{2}\right|\left(\left|w_{2}\right|+\left|w_{1}\right| t^{2 \beta-1}\right)}{|w| \Gamma(\alpha-\gamma+1)} \int_{0}^{T}(T-s)^{\alpha-\gamma}\left\|f\left(s, y_{n}(s)\right)-f(s, y(s))\right\| d s \\
& +\frac{\left|b_{2}\right|\left(\left|w_{2}\right|+\left|w_{1}\right| t^{2 \beta-1}\right)}{|w| \Gamma\left(\alpha-\gamma+q_{2}+1\right)} \int_{0}^{\eta_{2}}\left(\eta_{2}-s\right)^{\alpha-\gamma+q_{2}}\left\|f\left(s, y_{n}(s)\right)-f(s, y(s))\right\| d s \\
& \leq\left\{\frac{T^{\alpha-\gamma+1}}{\Gamma(\alpha+1)}+\frac{\left|b_{1}\right|\left(\left|w_{3}\right| T^{2 \beta-1}+\left|w_{4}\right|\right)}{|w| \Gamma\left(\alpha-\gamma+q_{1}+2\right)} \eta_{1}^{\alpha-\gamma+q_{1}+1}+\frac{\left|a_{2}\right|\left(\left|w_{2}\right|+\left|w_{1}\right| T^{2 \beta-1}\right)}{|w| \Gamma(\alpha-\gamma+2)} T^{\alpha-\gamma+1}\right. \\
& \left.+\frac{\left|b_{2}\right|\left(\left|w_{2}\right|+\left|w_{1}\right| T^{2 \beta-1}\right)}{|w| \Gamma\left(\alpha-\gamma+q_{2}+2\right)} \eta_{2}^{\alpha-\gamma+q_{2}+1}\right\}\left\|f\left(s, y_{n}(s)\right)-f(s, y(s))\right\| .
\end{aligned}
$$

Since $f$ is of Caratheodory type, then by the Lebesgue dominated convergence theorem, we have

$$
\left\|\aleph\left(y_{n}\right)-\aleph(y)\right\|_{\infty} \rightarrow 0 \text { as } n \rightarrow \infty
$$

Step 2. We show that $\aleph$ maps $D$ into $D$. 
Take $y \in D, t \in J$ and assume that $\aleph y(t) \neq 0$.

$$
\begin{aligned}
& \left\|t^{1-\gamma}(\aleph y)(t)\right\| \leq t^{1-\gamma}\left[I^{\alpha} f(s, y(s))(t)+\frac{\left(\left|w_{3}\right| t^{\gamma+2 \beta-2}+\left|w_{4}\right| t^{\gamma-1}\right)}{|w|}\left|b_{1}\right| I^{\alpha-\gamma+q_{1}+1} f(s, y(s))\left(\eta_{1}\right)\right. \\
& +\frac{\left(\left|w_{2}\right| t^{\gamma-1}+\left|w_{1}\right| t^{\gamma+2 \beta-2}\right)}{|w|}\left(\left|a_{2}\right| I^{\alpha-\gamma+1} f(s, y(s))(T)+\left|b_{2}\right| I^{\alpha-\gamma+q_{2}+1} f(s, y(s))\left(\eta_{2}\right)\right) \\
& \left.+\frac{t^{\gamma-1}}{|w|}\left(\left|w_{4} \lambda_{1}\right|+\left|w_{2} \lambda_{2}\right|\right)+\frac{t^{\gamma+2 \beta-2}}{|w|}\left(\left|w_{1} \lambda_{2}\right|+\left|w_{3} \lambda_{1}\right|\right)\right] \\
& \leq\left[t^{1-\gamma} I^{\alpha}|f(s, y(s))(t)|+\frac{\left|b_{1}\right|\left(\left|w_{3}\right| t^{2 \beta-1}+\left|w_{4}\right|\right)}{|w|} I^{\alpha-\gamma+q_{1}+1}\left|f(s, y(s))\left(\eta_{1}\right)\right|\right. \\
& +\frac{\left(\left|w_{2}\right|+\left|w_{1}\right| t^{2 \beta-1}\right)}{|w|}\left(\left|a_{2}\right| I^{\alpha-\gamma+1}|f(s, y(s))(T)|+\left|b_{2}\right| I^{\alpha-\gamma+q_{2}+1}\left|f(s, y(s))\left(\eta_{2}\right)\right|\right) \\
& \left.+\frac{\left(\left|w_{4} \lambda_{1}\right|+\left|w_{2} \lambda_{2}\right|\right)}{|w|}+\frac{t^{2 \beta-1}}{|w|}\left(\left|w_{1} \lambda_{2}\right|+\left|w_{3} \lambda_{1}\right|\right)\right] \\
& \leq\left[T^{1-\gamma} I^{\alpha}\|y\| p(s)(T)+\frac{\left|b_{1}\right|\left(\left|w_{3}\right| T^{2 \beta-1}+\left|w_{4}\right|\right)}{|w|} I^{\alpha-\gamma+q_{1}+1}\|y\| p(s)\left(\eta_{1}\right)\right. \\
& \left.+\frac{\left(\left|w_{2}\right|+\left|w_{1}\right| T^{2 \beta-1}\right)}{|w|}\left(\left|a_{2}\right| I^{\alpha-\gamma+1} p(s)(T)+\left|b_{2}\right| I^{\alpha-\gamma+q_{2}+1}\|y\| p(s)\left(\eta_{2}\right)\right)\right] \\
& +\frac{T^{2 \beta-1}\left(\left|w_{1} \lambda_{2}\right|+\left|w_{3} \lambda_{1}\right|\right)+\left(\left|w_{4} \lambda_{1}\right|+\left|w_{2} \lambda_{2}\right|\right)}{|w|} \\
& \leq p^{*} R\left[\frac{T^{\alpha-\gamma+1}}{\Gamma(\alpha+1)}+\frac{\left|b_{1}\right|\left(\left|w_{3}\right| T^{2 \beta-1}+\left|w_{4}\right|\right)}{|w| \Gamma\left(\alpha-\gamma+q_{1}+2\right)} \eta_{1}^{\alpha-\gamma+q_{1}+1}+\frac{\left|a_{2}\right|\left(\left|w_{2}\right|+\left|w_{1}\right| T^{2 \beta-1}\right)}{|w| \Gamma(\alpha-\gamma+2)} T^{\alpha-\gamma+1}\right. \\
& \left.+\frac{\left|b_{2}\right|\left(\left|w_{2}\right|+\left|w_{1}\right| T^{2 \beta-1}\right)}{\left|w_{\mid}\right| \Gamma\left(\alpha-\gamma+q_{2}+2\right)} \eta_{2}^{\alpha-\gamma+q_{2}+1}\right]+\frac{T^{2 \beta-1}\left(\left|w_{1} \lambda_{2}-w_{3} \lambda_{1}\right|\right)+\left(\left|w_{4} \lambda_{1}\right|+\left|w_{2} \lambda_{2}\right|\right)}{|w|} \\
& =p^{*} R L+\frac{T^{2 \beta-1}\left(\left|w_{1} \lambda_{2}\right|+\left|w_{3} \lambda_{1}\right|\right)+\left(\left|w_{4} \lambda_{1}\right|+\left|w_{2} \lambda_{2}\right|\right)}{|w|} \leq R .
\end{aligned}
$$

Next, we show that $\aleph(D)$ is equicontinuous. By Step 2, it is obvious that $\aleph(D) \subset C_{1-\gamma}(J, E)$ is bounded. For the equicontinuity of $\aleph(D)$, let $t_{1}, t_{2} \in J, t_{1}<t_{2}$ and $y \in D$, so $t_{2}^{1-\gamma} \aleph y\left(t_{2}\right)-t_{1}^{1-\gamma} \aleph y\left(t_{1}\right) \neq 0$. Hence,

$$
\begin{aligned}
& \left\|t_{2}^{1-\gamma} \aleph y\left(t_{2}\right)-t_{1}^{1-\gamma} \aleph y\left(t_{1}\right)\right\| \leq I^{\alpha}\left(t_{2}^{1-\gamma} f(s, x(s))\left(t_{2}\right)-t_{1}^{1-\gamma} f(s, x(s))\left(t_{1}\right)\right. \\
& +\frac{\left|b_{1} w_{3}\right|\left(t_{2}^{2 \beta-1}-t_{1}^{2 \beta-1}\right)}{|w|} I^{\alpha-\gamma+q_{1}+1} f(s, y(s))\left(\eta_{1}\right)+\left|w_{1}\right| \frac{\left(t_{1}^{2 \beta-1}-\mid t_{2}^{2 \beta-1}\right)}{|w|}\left(\left|a_{2}\right| I^{\alpha-\gamma+1} f(s, y(s))(T)\right. \\
& \left.+\left|b_{2}\right| I^{\alpha-\gamma+q_{2}+1} f(s, y(s))\left(\eta_{2}\right)\right)+\frac{t_{2}^{2 \beta-1}-t_{1}^{2 \beta-1}}{|w|}\left(\left|w_{1} \lambda_{2}-w_{3} \lambda_{1}\right|\right) \\
& \leq \frac{p^{*} R}{\Gamma(\alpha)}\left[t_{2}^{1-\gamma} \int_{0}^{t_{1}}\left(t_{2}-s\right)^{\alpha-1} d s-t_{1}^{1-\gamma} \int_{0}^{t_{1}}\left(t_{1}-s\right)^{\alpha-1} d s+t_{2}^{1-\gamma} \int_{t_{1}}^{t_{2}}\left(t_{2}-s\right)^{\alpha-1} d s\right] \\
& +p^{*} R\left[\frac{\left|w_{3} b_{1}\right|\left(t_{2}^{2 \beta-1}-t_{1}^{2 \beta-1}\right)}{|w|} I^{\alpha-\gamma+q_{1}+1}(1)\left(\eta_{1}\right)\right. \\
& \left.+\frac{\left|w_{1}\right|\left(t_{1}^{2 \beta-1}-t_{2}^{2 \beta-1}\right)}{|w|}\left(\left|a_{2}\right| I^{\alpha-\gamma+1}(1)(T)+\left|b_{2}\right| I^{\alpha-\gamma+q_{2}+1}(1)\left(\eta_{2}\right)\right)\right]+\frac{t_{2}^{2 \beta-1}-t_{1}^{2 \beta-1}}{|w|}\left(\left|w_{1} \lambda_{2}-w_{3} \lambda_{1}\right|\right) \\
& \leq p^{*} R\left[\frac{\left(t_{2}^{\alpha-\gamma+1}-t_{1}^{\alpha-\gamma+1}\right)}{\Gamma(\alpha+1)}+\frac{\left|b_{1} w_{3}\right|\left(t_{2}^{2 \beta-1}-t_{1}^{2 \beta-1}\right)}{|w| \Gamma\left(\alpha-\gamma+q_{1}+2\right)} \eta_{1}^{\alpha-\gamma+q_{1}+1}\right. \\
& \left.+\frac{\left|w_{1}\right|\left(t_{1}^{2 \beta-1}-t_{2}^{2 \beta-1}\right)}{|w|}\left(\frac{\left|a_{2}\right| T^{\alpha-\gamma+1}}{\Gamma(\alpha-\gamma+2)}+\frac{\left|b_{2}\right| \eta_{2}^{\alpha-\gamma+q_{2}+1}}{\Gamma\left(\alpha-\gamma+q_{2}+2\right)}\right)\right]+\frac{t_{2}^{2 \beta-1}-t_{1}^{2 \beta-1}}{|w|}\left(\left|w_{1} \lambda_{2}-w_{3} \lambda_{1}\right|\right)
\end{aligned}
$$

As $t_{1} \rightarrow t_{2}$, the right hand side of the above inequality tends to zero. Hence $\aleph(D) \subset D$.

Step 3. The implication (9) holds.

Now let $V$ be a bounded and equicontinuous subset of $D$. Hence $t \mapsto v(t)=\mu(V(t))$ is continuous on $J$ such that $V \subset \overline{\mathrm{conv}}(0 \cup \aleph(V))$. Clearly, $V(t) \subset \overline{\mathrm{conv}}(\{0\} \cup \aleph(V))$ for all $t \in J$. Hence $\aleph V(t) \subset \aleph D(t), t \in J$ is bounded in $E$. By assumption (H3), and the properties of measure $\mu$, we have, for each $t \in J$, 


$$
\begin{aligned}
t^{1-\gamma} v(t) \leq & \left.\mu\left(t^{1-\gamma} N(V)(t) \cup\{0\}\right)\right) \leq \mu\left(t^{1-\gamma}(N V)(t)\right) \\
\leq & \mu\left\{t ^ { 1 - \gamma } \left[I^{\alpha} f(t, V(t))+\frac{\left(w_{3} t^{\gamma+2 \beta-2}-w_{4} t^{\gamma-1}\right)}{w} b_{1} I^{\alpha-\gamma+q_{1}+1} f(s, V(s))\left(\eta_{1}\right)+\frac{t^{\gamma-1}}{w}\left(w_{4} \lambda_{1}-w_{2} \lambda_{2}\right)\right.\right. \\
& +\frac{a_{2}\left(w_{2} t^{\gamma-1}-w_{1} t^{\gamma+2 \beta-2}\right)}{w} I^{\alpha-\gamma+1} f(s, V(s))(T)+\frac{b_{2}\left(w_{2} t^{\gamma-1}-w_{1} t^{\gamma+2 \beta-2}\right)}{w} I^{\alpha-\gamma+q_{2}+1} f(s, V(s))\left(\eta_{2}\right) \\
& \left.\left.+\frac{t^{\gamma+2 \beta-2}}{w}\left(w_{1} \lambda_{2}-w_{3} \lambda_{1}\right)\right]\right\} \\
\leq & t^{1-\gamma} I^{\alpha} \mu(f(s, V(s)))(t)+\frac{\left|b_{1}\right|\left(\left|w_{3}\right| t^{2 \beta-1}+\left|w_{4}\right|\right)}{|w|} I^{\alpha-\gamma+q_{1}+1} \mu(f(s, V(s)))\left(\eta_{1}\right) \\
& +\frac{\left|a_{2}\right|\left(\left|w_{2}\right|+\left|w_{1}\right| t^{2 \beta-1}\right)}{|w|} I^{\alpha-\gamma+1} \mu(f(s, V(s)))(T)+\frac{\left|b_{2}\right|\left(\left|w_{2}\right|+\left|w_{1}\right| t^{2 \beta-1}\right)}{|w|} I^{\alpha-\gamma+q_{2}+1} \mu(f(s, V(s)))\left(\eta_{2}\right) \\
\leq & t^{1-\gamma} I^{\alpha}(p(s) v(s))(t)+\frac{\left|b_{1}\right|\left(\left|w_{3}\right| T^{2 \beta-1}+\left|w_{4}\right|\right)}{|w|} I^{\alpha-\gamma+q_{1}+1}(p(s) v(s))\left(\eta_{1}\right) \\
& +\frac{\left|a_{2}\right|\left(\left|w_{2}\right|+\left|w_{1}\right| T^{2 \beta-1}\right)}{|w|} I^{\alpha-\gamma+1}(p(s) v(s))(T)+\frac{\left|b_{2}\right|\left(\left|w_{2}\right|+\left|w_{1}\right| T^{2 \beta-1}\right)}{|w|} I^{\alpha-\gamma+q_{2}+1}(p(s) v(s))\left(\eta_{2}\right) \\
\leq & p^{*}\|v\|_{\infty}\left[T^{1-\gamma} I^{\alpha}(1)(T)+\frac{\left|b_{1}\right|\left(\left|w_{3}\right| T^{2 \beta-1}+\left|w_{4}\right|\right)}{|w|} I^{\alpha-\gamma+q_{1}+1}(1)\left(\eta_{1}\right)\right. \\
& \left.+\frac{\left|a_{2}\right|\left(\left|w_{2}\right|+\left|w_{1}\right| T^{2 \beta-1}\right)}{|w|} I^{\alpha-\gamma+1}(1)(T)+\frac{\left|b_{2}\right|\left(\left|w_{2}\right|+\left|w_{1}\right| T^{2 \beta-1}\right)}{|w|} I^{\alpha-\gamma+q_{2}+1}(1)\left(\eta_{2}\right)\right] \\
\leq & p^{*}\|v\|_{\infty}\left[\frac{T^{\alpha-\gamma+1}}{\Gamma(\alpha+1)}+\frac{\left|b_{1}\right|\left(\left|w_{3}\right| T^{2 \beta-1}+\left|w_{4}\right|\right)}{|w| \Gamma\left(\alpha-\gamma+q_{1}+2\right)} \eta_{1}^{\alpha-\gamma+q_{1}+1}+\frac{\left|a_{2}\right|\left(\left|w_{2}\right|+\left|w_{1}\right| T^{2 \beta-1}\right)}{|w| \Gamma(\alpha-\gamma+2)} T^{\alpha-\gamma+1}\right. \\
& \left.+\frac{\left|b_{2}\right|\left(\left|w_{2}\right|+\left|w_{1}\right| T^{2 \beta-1}\right)}{|w| \Gamma\left(\alpha-\gamma+q_{2}+2\right)} \eta_{2}^{\alpha-\gamma+q_{2}+1}\right] \\
= & p^{*}\|v\|_{\infty} L .
\end{aligned}
$$

which gives $\|v\|_{\infty}\left(1-p^{*} L\right) \leq 0$. From (17), we get $\|v\|=0$, that is, $v(t)=\mu(V(t))=0$, for each $t \in J$. Then $V$ is relatively compact in $E$. In view of the Ascoli-Arzela theorem, $V$ is relatively compact in $D$. Applying now Theorem 1 , we conclude that $\aleph$ has a fixed point which is a solution of (1).

\section{Example}

Example 1. Let us consider the following Hilfer fractional boundary value problem;

$$
\begin{cases}D_{0^{+}}^{\frac{3}{2}, \frac{2}{3}} y(t)=f(t, y(t)), & t \in J:=[0,1] \\ D^{\frac{1}{6}} y(0)+I^{\frac{1}{3}} y\left(\frac{1}{3}\right)=1, & 0<q_{1} \leq 1 \\ D^{\frac{1}{6}} y(1)+I^{\frac{1}{6}} y\left(\frac{2}{3}\right)=2, & 0<q_{2} \leq 1\end{cases}
$$

where $\alpha=\frac{3}{2}, \quad \beta=\frac{2}{3}, \quad \gamma=\frac{7}{6}, T=1, a_{1}=a_{2}=1, b_{1}=b_{1}=1, \lambda_{1}=1, \lambda_{2}=2, q_{2}=\frac{1}{6}, q_{1}=\frac{1}{2}, \eta_{1}=\frac{1}{3}$, $\eta_{2}=\frac{2}{3}$.

Let $E=l^{1}=\left\{x=\left(x_{1}, x_{2}, \ldots, x_{n}, \ldots\right): \sum_{n=1}^{\infty}\left|x_{n}\right|<\infty\right\}$ with the norm $\left\|y_{n}\right\|_{E}=\sum_{n=1}^{\infty}\left|x_{n}\right|$. Set $y=$ $\left(y_{1}, y_{2}, \ldots, y_{n}, \ldots\right), f=\left(f_{1}, f_{2}, \ldots, f_{n}, \ldots\right)$, with $\left.f(t, y t)\right)=\frac{1}{e^{t+2}}\left|y_{n}(t)\right|, t \in J$. Clearly, the function $f$ is continuous. For each $y_{n} \in \mathbb{R}$ and $t \in J$, we have $\|f(t, y(t))\| \leq \frac{1}{e^{t+2}}\left\|y_{n}\right\|$. Hence conditions (H1), (H2) and (H3) hold with $p(t)=\frac{1}{e^{t+2}}, t \in J$ and (H3) is satisfied with $p^{*}=e^{-2}$. Now, we can find that $p^{*} L \simeq \frac{693}{2500} \leq 1$, hence (H4) is satisfied and we have $p^{*} R L+K \leq R$. Thus $R>\frac{K}{1-L p^{*}}$, so $R>\frac{10053}{2000}$. Consequently, Theorem 3 implies that Problem (19) has a solution defined on $J$.

\section{Conclusions}

In this paper, we consider the existence of solutions of the boundary value problem for a nonlinear fractional differential equation. Several existence and uniqueness results have been derived by using a method involving a measure of noncompactness and a fixed point theorem of Mönch type. Our results are quite general 
and give rise to many new cases by assigning different values to the parameters involved in the problem. For explanation, we enlist some special cases.

In case we choose $a_{1}=a_{2}=T=\beta=1, b_{1}=b_{2}=-1$ and $\lambda_{1}=\lambda_{2}=0$ the Problem (1) reduces to the case considered in [26] in the scalar case using the standard tools of fixed point theory and Leray-Schauder nonlinear alternative. Here we extend the results of [26] to cover the abstract case. We remark the cases when considered in conclusion in [26] also exist here.

Author Contributions: All authors contributed equally to the writing of this paper. All authors read and approved the final manuscript.

Conflicts of Interest: "The authors declare no conflict of interest."

\section{References}

[1] Hilfer, R. (2000). Applications of fractional calculus in physics. Singapore, World scientific.

[2] Kamocki, R., \& Obczynski, C. (2016). On fractional Cauchy-type problems containing Hilfer's derivative. Electronic Journal of Qualitative Theory of Differential Equations, 2016(50), 1-12.

[3] Abbas, S., Benchohra, M., \& N'Guerekata, G. M. (2014). Advanced fractional differential and integral equations. Nova Science Publishers.

[4] Samko, S. G., Kilbas, A. A., \& Marichev, O. I. (1993). Fractional integrals and derivatives (Vol. 1). Yverdon-les-Bains, Switzerland: Gordon and Breach Science Publishers, Yverdon.

[5] Kilbas, A. A., Srivastava, H. M., \& Trujillo, J. J. (2006). Theory and applications of fractional differential equations (Vol. 204). elsevier.

[6] Yong, Z. (2014). Basic Theory Of Fractional Differential Equations (Vol. 6). World Scientific.

[7] Abbas, S., Benchohra, M., Henderson, J., \& Lazreg, J. E. (2017). Measure of noncompactness and impulsive Hadamard fractional implicit differential equations in Banach spaces. Mathematics in Engineering, Science \& Aerospace (MESA), $8(3), 1-19$.

[8] Abbas, S., Benchohra, M., Lazreg, J. E., \& Zhou, Y. (2017). A survey on Hadamard and Hilfer fractional differential equations: analysis and stability. Chaos, Solitons \& Fractals, 102, 47-71.

[9] Abbas, S., Benchohra, M., Lazreg, J. E., \& Nieto, J. J. (2018). On a coupled system of Hilfer and Hilfer \# Hadamard fractional defferential equation in Banach spaces. Journal of Nonlinear Functional Analysis, Article ID 12.

[10] Hilfer, R., Luchko, Y., \& Tomovski, Z. (2009). Operational method for the solution of fractional differential equations with generalized Riemann-Liouville fractional derivatives. Fractional Calculus and Applied Analysis, 12(3), 299-318.

[11] Bhairat, S. P. (2019). Existence and continuation of solutions of Hilfer fractional differential equations. Journal of Mathematical Modeling, 7(1), 1-20.

[12] Vivek, D., Kanagarajan, K., \& Elsayed, E. M. (2018). Nonlocal initial value problems for implicit differential equations with Hilfer-Hadamard fractional derivative. Nonlinear Analysis: Modelling and Control, 23(3), 341-360.

[13] Wang, J., \& Zhang, Y. (2015). Nonlocal initial value problems for differential equations with Hilfer fractional derivative. Applied Mathematics and Computation, 266, 850-859.

[14] Yang, M., \& Wang, Q. R. (2017). Approximate controllability of Hilfer fractional differential inclusions with nonlocal conditions. Mathematical Methods in the Applied Sciences, 40(4), 1126-1138.

[15] Agarwal, R. P., Meehan, M., \& O'regan, D. (2001). Fixed point theory and applications (Vol. 141). Cambridge university press.

[16] Bana, J., \& Goebel, K. (1980). Measures of noncompactness in Banach spaces. Lecture Notes in Pure and Applied Mathematics, 60, 97 pages.

[17] Banas, J., Jleli, M., Mursaleen, M., Samet, B., \& Vetro, C. (Eds.). (2017). Advances in nonlinear analysis via the concept of measure of noncompactness. Springer Singapore.

[18] Benchohra, M., Henderson, J., \& Seba, D. (2008). Measure of noncompactness and fractional differential equations in Banach spaces. Communications in Applied Analysis, 12(4), 419-428.

[19] Akhmerov, R. R., Kamenskii, M. I., Potapov, A. S., Rodkina, A. E., \& Sadovskii, B. N. (1992). Measures of noncompactness and condensing operators (Vol. 55). Basel, Birkhäuser.

[20] Alvárez, J. C. (1985). Measure of noncompactness and fixed points of nonexpansive condensing mappings in locally convex spaces. Revista de la Real Academia de Ciencias Exactas, Fisicas y Naturales (Espana), 79(1-2), 53-66.

[21] Mönch, H. (1980). Boundary value problems for nonlinear ordinary differential equations of second order in Banach spaces. Nonlinear Analysis: Theory, Methods \& Applications, 4(5), 985-999.

[22] Szufla, S. (1986). On the application of measure of noncompactness to existence theorems. Rendiconti del Seminario Matematico della Universita di Padova, 75, 1-14.

[23] Furati, K. M., Kassim, M. D., \& Tatar, N. E. (2013). Non-existence of global solutions for a differential equation involving Hilfer fractional derivative. Electronic Journal of Differential Equations, 2013(235), 1-10. 
[24] Kou, C., Liu, J., \& Ye, Y. (2010). Existence and uniqueness of solutions for the Cauchy-type problems of fractional differential equations. Discrete Dynamics in Nature and Society, 2010, Article ID 142175.

[25] Furati, K. M., \& Kassim, M. D. (2012). Existence and uniqueness for a problem involving Hilfer fractional derivative. Computers \& Mathematics with Applications, 64(6), 1616-1626.

[26] Ahmad, B., Ntouyas, S. K., \& Assolami, A. (2013). Caputo type fractional differential equations with nonlocal Riemann-Liouville integral boundary conditions. Journal of Applied Mathematics and computing, 41(1-2), 339-350.

[27] Ahmad, B., Ntouyas, S. K., Tariboon, J., \& Alsaedi, A. (2017). Caputo type fractional differential equations with nonlocal Riemann-Liouville and Erdélyi-Kober type integral boundary conditions. Filomat, 31(14), 4515-4529.

[28] Banas, J., \& Nalepa, R. (2016). On a measure of noncompactness in the space of functions with tempered increments. Journal of Mathematical Analysis and Applications, 435(2), 1634-1651.

[29] Banas, J., \& Olszowy, L. (2001). Measures of noncompactness related to monotonicity. Annales Societatis Mathematicae Polonae. Seria 1: Commentationes Mathematicae, 41, 13-23.

[30] Banas, J., \& Sadarangani, K. (2008). On some measures of noncompactness in the space of continuous functions. Nonlinear Analysis: Theory, Methods E Applications, 68(2), 377-383.

[31] Hamani, S., \& Benhamida, W. (2018). Measure of Noncompactness and Caputo-Hadamard Fractional Differential Equations in Banach Spaces. Eurasian Bulletin of Mathematics, 1(3), 98-106.

[32] Vivek, D., Kanagarajan, K., \& Sivasundaram, S. (2018). On the behavior of solutions of Hilfer\# Hadamard type fractional neutral pantograph equations with boundary conditions. Communications in Applied Analysis, 22(3), $211-232$.

[33] Dajun, G., Lakshmikantham, V., \& Xinzhi, L. (1996). Nonlinear integral equations in abstract spaces. The Netherlands: Kluwer Acadmic Publishers.

[34] Gu, H., \& Trujillo, J. J. (2015). Existence of mild solution for evolution equation with Hilfer fractional derivative. Applied Mathematics and Computation, 257, 344-354.

[35] Haddouchi, F. (2018). Existence results for a class of Caputo type fractional differential equations with Riemann-Liouville fractional integrals and Caputo fractional derivatives in boundary conditions. arXiv preprint arXiv:1805.06015.

(C) 2020 by the authors; licensee PSRP, Lahore, Pakistan. This article is an open access article distributed under the terms and conditions of the Creative Commons Attribution (CC-BY) license (http://creativecommons.org/licenses/by/4.0/). 